\title{
Correlation between therapy response assessment using FDG PET/CT and histopathologic tumor regression grade in hepatic metastasis of colorectal carcinoma after neoadjuvant therapy
}

Burger, Irene A ; Schwarz, Esther I ; Samarin, Andrei ; Breitenstein, Stefan ; Weber, Achim ; Hany, Thomas F

\begin{abstract}
PURPOSE: To evaluate the correlation between change in FDG uptake before and after chemotherapy in hepatic metastases of colorectal carcinoma (HCRC) and a histopathologic tumor regression grade (TRG). METHODS: In patients with HCRC, PET/CT data prior to hepatic surgery were retrospectively analyzed under an IRB waiver. The maximum standard uptake value $(\mathrm{SUV}(\max ))$ was measured before and after chemotherapy. The relative change of FDG activity in the identified lesions was calculated (dSUV). Histopathological specimens of resected metastases were graded on a 5-score TRG scale. A TRG of 1-3 was rated as a responding to therapy, whereas TRG 4-5 were regarded as non-responding lesions. RESULTS: 31 lesions were identified in 23 patients. Mean SUV(max) before and after therapy was $6.9 \pm 3.7$ and $3.5 \pm 1.8$, respectively. The area under the receiver operator characteristic curve revealed a conclusive correlation between TRG and dSUV (AUC 0.773; $95 \%$ confidence interval 0.599-0.946) with a cut off at $41 \%$ decrease in FDG activity yielding a sensitivity and specificity of 72 and $75 \%$, respectively. CONCLUSION: A relative change in FDG activity (dSUV) of more than $41 \%$ decrease correlated significantly with histopathological tumor regression and might be a prognostic tool for response to chemotherapy in HCRC.
\end{abstract}

DOI: https://doi.org/10.1007/s12149-012-0670-8

Posted at the Zurich Open Repository and Archive, University of Zurich

ZORA URL: https://doi.org/10.5167/uzh-69442

Journal Article

Published Version

Originally published at:

Burger, Irene A; Schwarz, Esther I; Samarin, Andrei; Breitenstein, Stefan; Weber, Achim; Hany, Thomas F (2013). Correlation between therapy response assessment using FDG PET/CT and histopathologic tumor regression grade in hepatic metastasis of colorectal carcinoma after neoadjuvant therapy. Annals of Nuclear Medicine, 27(2):177-183.

DOI: https://doi.org/10.1007/s12149-012-0670-8 


\title{
Correlation between therapy response assessment using FDG PET/CT and histopathologic tumor regression grade in hepatic metastasis of colorectal carcinoma after neoadjuvant therapy
}

\author{
Irene A. Burger $\cdot$ Esther I. Schwarz • \\ Andrei Samarin - Stefan Breitenstein • \\ Achim Weber • Thomas F. Hany
}

Received: 4 September 2012 / Accepted: 18 November 2012/Published online: 12 December 2012

(C) The Japanese Society of Nuclear Medicine 2012

\begin{abstract}
Purpose To evaluate the correlation between change in FDG uptake before and after chemotherapy in hepatic metastases of colorectal carcinoma (HCRC) and a histopathologic tumor regression grade (TRG).

Methods In patients with HCRC, PET/CT data prior to hepatic surgery were retrospectively analyzed under an IRB waiver. The maximum standard uptake value $\left(\mathrm{SUV}_{\max }\right)$ was measured before and after chemotherapy. The relative change of FDG activity in the identified lesions was calculated (dSUV). Histopathological specimens of resected metastases were graded on a 5-score TRG scale. A TRG of 1-3 was rated as a responding to therapy, whereas TRG 4-5 were regarded as non-responding lesions.

Results 31 lesions were identified in 23 patients. Mean SUV $_{\text {max }}$ before and after therapy was $6.9 \pm 3.7$ and $3.5 \pm 1.8$, respectively. The area under the receiver operator characteristic curve revealed a conclusive correlation between TRG and dSUV (AUC 0.773; $95 \%$ confidence interval 0.599-0.946) with a cut off at $41 \%$ decrease in FDG activity yielding a sensitivity and specificity of 72 and $75 \%$, respectively.
\end{abstract}

I. A. Burger $(\bowtie) \cdot$ A. Samarin · T. F. Hany

Division of Nuclear Medicine, Department Medical Radiology,

University Hospital Zurich, Ramistr. 100, 8091 Zurich,

Switzerland

e-mail: irene.burger@usz.ch

E. I. Schwarz - A. Weber

Institute of Surgical Pathology, Department of Pathology,

University Hospital Zurich, Zurich, Switzerland

S. Breitenstein

Department of Visceral Surgery, University Hospital Zurich,

Zurich, Switzerland
Conclusion A relative change in FDG activity (dSUV) of more than $41 \%$ decrease correlated significantly with histopathological tumor regression and might be a prognostic tool for response to chemotherapy in HCRC.

Keywords FDG PET/CT - Tumor regression grade Colorectal cancer - Hepatic metastasis - Therapy response assessment

\section{Introduction}

In the past decades the use of neoadjuvant chemotherapy before surgery for hepatic metastasis of colorectal carcinoma (HCRC) gained significance [1]. Thanks to the introduction of more effective chemotherapeutic agents and targeted agents for HCRC this approach improved the outcome [2]. The main intention thereby is to render primarily unresectable patients resectable [3, 4]. To determine the eligibility of patients preoperative ${ }^{18} \mathrm{~F}$-fluorodeoxyglucose (FDG) PET/CT has shown to reduce futile laparotomies since not only the liver but the entire body is evaluated and distant recurrences or progression can be detected [5].

Metabolic alterations in tumor cells have been shown to occur before alterations in tumor size and might be a better indicative of tumor response to therapy [6]. Therefore, the preoperative FDG PET/CT scan has also been used for therapy response assessment [7]. For evaluation of tumor response with FDG PET, the European Organization for Research and Treatment of Cancer (EORTC) released a widely accepted standardization in 1999 [8]. However, since then different percentages of relative change in FDG uptake have been suggested, to distinguish complete or partial remission from stable or progressive disease. 
EORTC guidelines suggested a partial remission with $>15 \%$ decrease in SUV after one cycle of chemotherapy, or $>25 \%$ decrease after more than one cycle [8], others a decrease of $>30 \%$ for partial remission [9].

These generalized guidelines for all solid tumor entities lack pathological correlates and cannot take variable biological tumor behaviour into account. Therefore, a correlation of the change in FDG measured as the relative change in $\mathrm{SUV}_{\max }$ with histopathological tumor regression grades (TRGs) for specific tumor types has been suggested [10-13]. These studies showed that for locally advanced rectal cancer a decrease between 53 and $66 \%$ in FDG activity correlated well with a good pathological response to chemo radiation therapy $[10,13]$. On the other hand, the correlation between decrease in FDG uptake and histopathological response in esophageal carcinomas was not significant $[11,12,14]$.

Histopathological analysis of residual tumor viability with a TRG was initially proposed for rectal cancer, reaching from Grade 1 with complete fibrosis to Grade 5 with complete absence of regressive changes [15]. A modified version of this TRG scoring system has been suggested to assess the therapy response in HCRC [16]. With this score TRG responders had a significantly improved disease-free survival (DFS) and overall survival (OS) [16].

To our knowledge no previous study investigated the correlation between TRG, and the relative change in FDG uptake before and after chemotherapy for HCRC. The aim of our analysis was to correlate the relative change in FDG uptake with TRG and to determine the optimal cut off to distinguish histopathological responders from nonresponders with FDG PET/CT.

\section{Materials and methods}

Patients

We consecutively screened all patients between June 2007 and July 2010 with colorectal carcinoma referred from the visceral surgery department to the division of nuclear medicine and selected those patients with HCRC who underwent FDG PET/CT before and after chemotherapy. Patients were eligible if the second FDG PET/CT was within 2-7 weeks after last chemotherapy. 69 patients met the inclusion criteria. 3 patients had partial liver resection between both scans and 2 patients had one FDG PET/CT examination in a different institution and were therefore excluded. Of the remaining 64 patients, 42 had surgery with resection of hepatic metastases within 8 weeks after FDG PET/CT [17, 18]. From the medical records, a clear correlation between pathology and FDG $\mathrm{PET} / \mathrm{CT}$ was possible for 31 lesions $(\max 3$ lesions per patient) in 23 patients (Fig. 1).

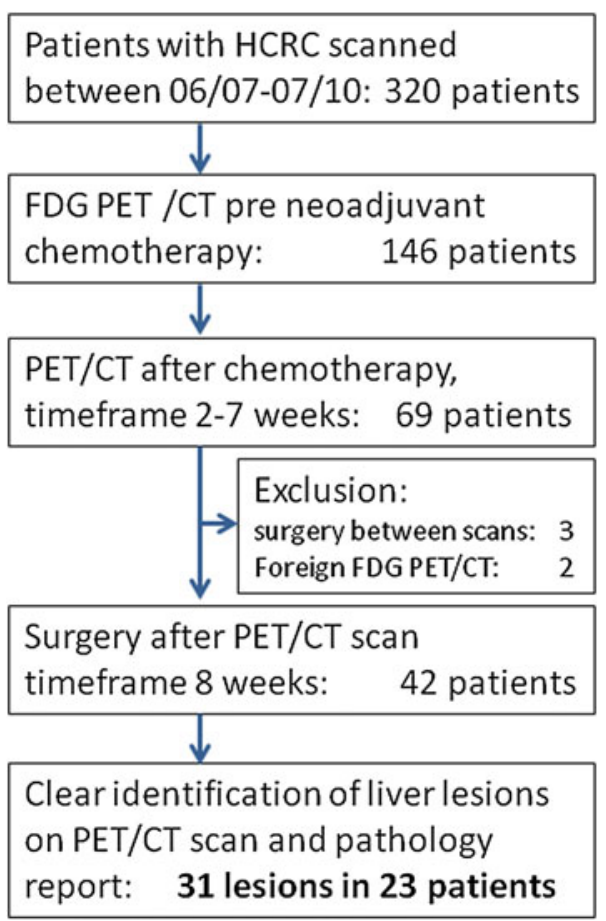

Fig. 1 Flow chart illustrating the eligibility criteria for patient and lesion selection

PET/CT acquisition and analysis

All patients were examined using a routine clinical protocol on dedicated PET/CT scanners (GE Healthcare DSTX, 16- or 64-slices CT, 7-8 frames, frame time 1.5 or $2 \mathrm{~min}$ ) with injection of $350 \mathrm{MBq}$ FDG 45-60 min before examination. A low dose unenhanced CT-scan was performed for attenuation correction and used for anatomical localization $(80 \mathrm{~mA}, 140 \mathrm{kV})$. Approximate total dose equivalent for the entire PET/CT examination $=10 \mathrm{mSv}$. To reduce bladder activity patients had to urinate just before image acquisition [19].

Image analysis was performed by a dual board certified nuclear medicine physician and radiologist (TFH), blinded for the results from histopathology. The activity in the identified lesions was measured as the maximum value in a volume of interest (VOI) around the whole selected lesion. The relative change in FDG activity between the two PET/ CT scans before and after neo-adjuvant (for resectable patients) or downstaging (for unresectable patients) chemotherapy was calculated (dSUV), Eq (1):

$$
\operatorname{dSUV}(\%)=\frac{\operatorname{SUV}_{\max }(\operatorname{scan} 2)-\operatorname{SUV}_{\max }(\operatorname{scan} 1)}{\operatorname{SUV}_{\max }(\operatorname{scan} 1)} \times 100 .
$$


Histopathological analysis

Histopathological specimens of resected metastases were evaluated for the proportion of viable tumor cells, tumor necrosis, and fibrosis in Hematoxylin and Eosin staining. TRG was graded on a 5-point scale based on the histological tumor response assessment described by RubbiaBrandt et al. [16]. TRG 1 signifies complete tumor regression showing only fibrosis as sign of regression and no residual cancer in the former metastasis. The amount of viable tumor cells increases with TRG whereas the extent of fibrosis decreases with complete absence in TRG 5.

\section{Statistical analysis}

The distribution of dSUV values for each histopathological TRG was analyzed using box plots. Correlation between TRG and dSUV was calculated with Pearson correlation. A

Table 1 Patient demographics

\begin{tabular}{ll}
\hline Number of patients & 23 \\
\hline Age (years \pm SD) & $57.2 \pm 8$ \\
Gender (male/female) & $(14 / 9)$ \\
Lesions identified & 31 \\
Time delay between & \\
Chemotherapy and PET/CT II (days \pm SD) & $25.3 \pm 21.7$ \\
PET/CT II and surgery (days \pm SD) & $27.6 \pm 25.7$ \\
Chemotherapy regimens (patients) & \\
FOLFOX & 12 \\
FOLFIRI (+ optional Bevacizumab) & 7 \\
5-FU (+ optional Bevacizumab or oxaliplatin) & 4 \\
Chemotherapy intention & 11 \\
Neo-adjuvant & 12 \\
Down staging &
\end{tabular}

Standard deviation (SD), folic acid-fluorouracil-oxaliplatin (FOLFOX), folic acid-fluorouracil-irinotecan (FOLFIRI), fluorouracil (5-FU)

Table 2 FDG PET/CT values: pre- and post-chemotherapy receiver operator characteristic (ROC) curve was generated for dSUV and TRG responders versus non-responders. The area under the curve (AUC) was calculated and the optimal cut off point for the ROC curve determined using the Youden index ( $J$ value), calculated as the maximum of $J=\mathrm{SN}+\mathrm{SP}-1$, where $\mathrm{SN}$ is sensitivity and $\mathrm{SP}$ is specificity, for each cut off volume [20].

\section{Results}

31 resected liver lesions were identified in 23 patients. 19 of 23 patients were treated with folic acid and fluorouracil combined with either oxaliplatin or irinotecan. 4 patients were treated with fluorouracil and optional bevacizumab or oxaliplatin. Demographic details are listed in Table 1.

In the baseline study the HCRC had SUV $\max$ values of $7.4 \pm 3.7$ (range 2.9-15.3). The $\mathrm{SUV}_{\max }$ for all lesions in the second scan was $3.5 \pm 2.1$ (range 1.6-12).

The histopathological analysis revealed complete fibrosis in 2 lesions (TRG 1), more fibrosis than residual viable tumor in 18 lesions (TRG 2 and 3) and more viable tumor than fibrosis in 11 lesions (TRG 4 and 5) (Table 2).

The relative change in $\mathrm{SUV}_{\text {max }}$ for the five histological TRG grades is given in Fig. 2 with box plots. The correlation between TRG and dSUV was significant (correlation $r=0.48, p=0.001,2$-tailed). The ROC curve revealed an AUC of 0.773 (95\% confidence interval 0.599-0.946), with an optimal cut off to distinguish responders from non-responders of dSUV $-41 \%$, yielding a sensitivity, specificity, positive and negative predictive value and an accuracy of 72, 75, 83, 62 and $74 \%$, respectively (Fig. 3).

With a cut off at dSUV $-41 \%, 15$ lesions were regarded responding in FDG PET/CT CT and also histopathological responders (TRG 1-3). Figure 4 is an example of complete remission in PET/CT with no residual FDG uptake and a complete histological tumor regression (TRG 1). 13 lesions were non-responders in FDG PET/CT, histopathology however revealed response to therapy in 5 of these lesions (all lesions TRG 3). The mean dSUV overall was $-41 \pm 42 \%$ (range -89.5 to $126 \%$ ). All lesions with

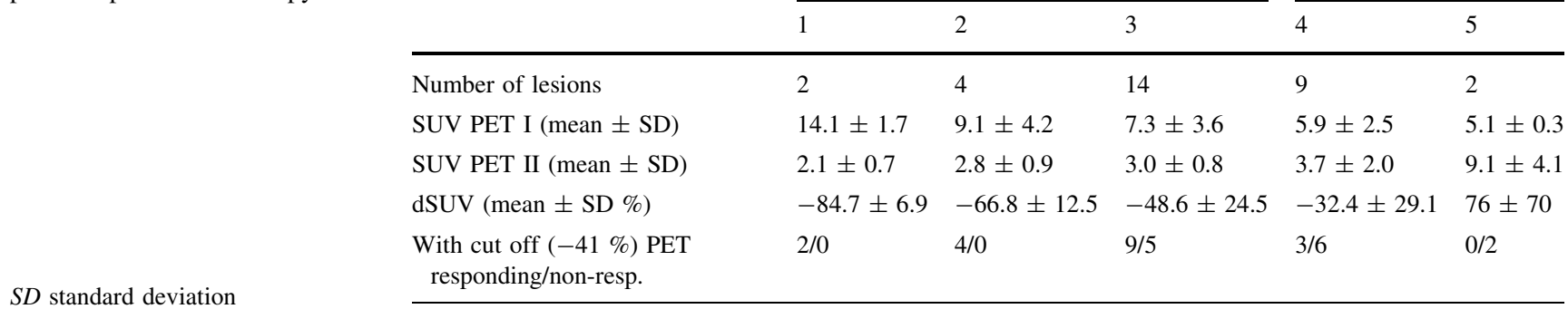




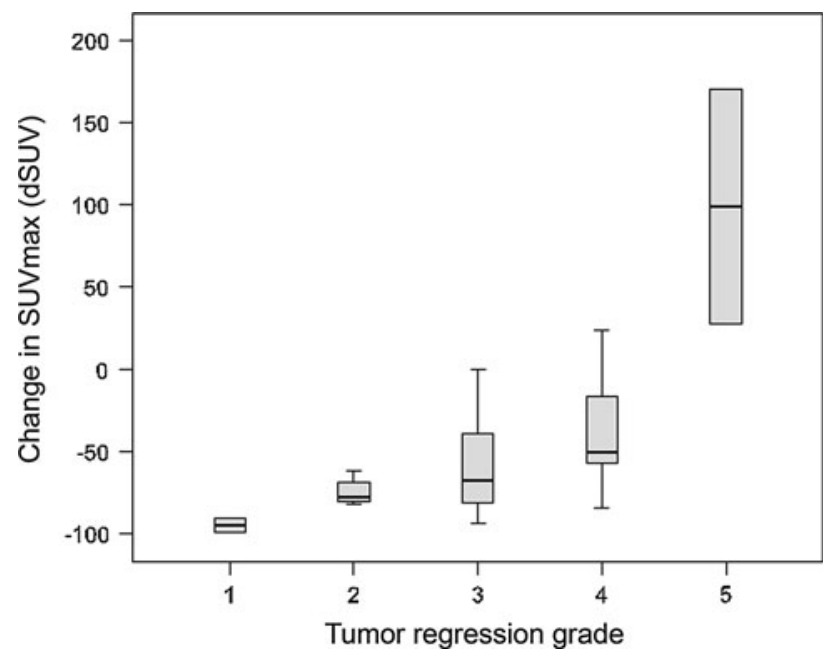

Fig. 2 Box plot illustration of the distribution of dSUV for the 5 different TRG groups

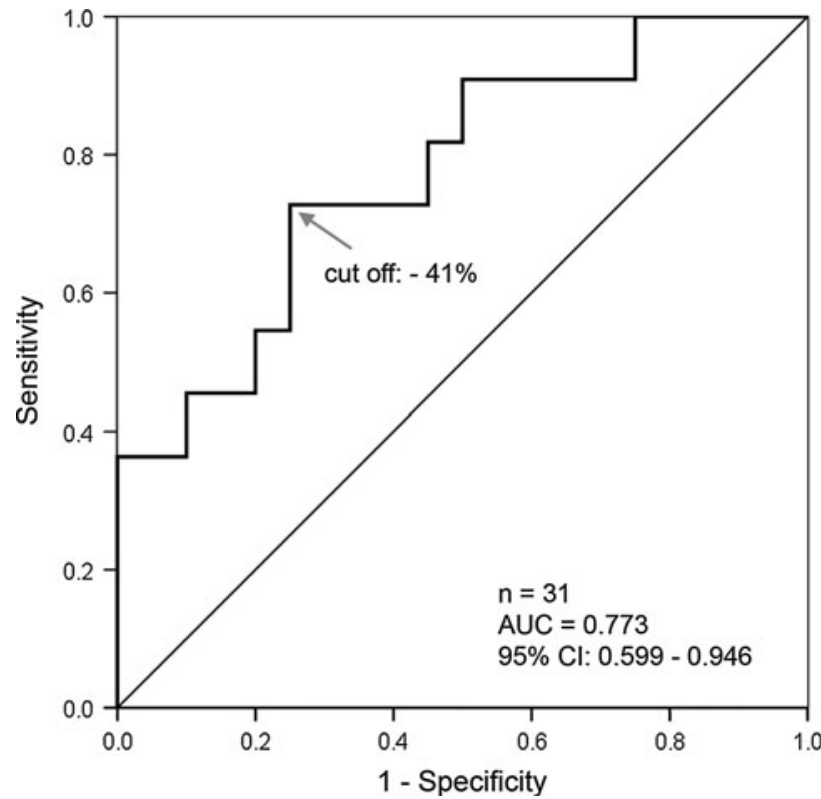

Fig. 3 Receiver operating characteristic curves for the change in SUV (dSUV) if TRG 1-3 are interpreted as responders, and TRG 4 and 5 as non-responders. The resulting cut off for dSUV is a decrease in activity of $41 \%$, yielding a sensitivity, specificity, positive and negative predictive value and an accuracy of $72,75,83,62$ and $74 \%$, respectively

an increase in dSUV showed no signs of histopathological response (TRG 4 or 5) (Table 3). Figure 5 illustrates a case of increased FDG uptake in the second PET/CT, corresponding to a high degree of remaining vital tumor cells analogous to TRG 5. In one lesion PET/CT suggested therapy response (dSUV) $-76 \%$; histopathology revealed a mixed image with areas of extended necrosis (Fig. $6 \mathrm{C}_{1}$ ), and areas of more vital cells than fibrosis (TRG 4, Fig. $6 \mathrm{C}_{2}$ ).

For six patients two lesions were included in the study, of those two patients had a decrease in dSUV of less than $41 \%$ in both lesions and were regarded as non-responders, whereas four patients decreased more than $41 \%$ in both lesions. In one patient 3 lesions could be identified on PET/ CT and pathology reports and were included in the study: two lesions decreased less than $41 \%$ and one lesion decreased $51 \%$ between the two PET/CT scans, resulting in a mixed response.

\section{Discussion}

The objective of this study was to assess the diagnostic accuracy of response assessment with FDG PET/CT in patients with HCRC treated with neoadjuvant chemotherapy by correlating change in FDG uptake with histopathological tumor response.

Overall, change in FDG activity in HCRC after chemotherapy correlated significant with overall survival in several studies and FDG PET seems to be a reliable prognostic tool to predict the efficacy of preoperative chemotherapy $[18,21]$.

Previous recommendations for therapy assessment with FDG PET/CT suggested a decrease of 15-30\% for a partial metabolic response $[8,9]$. In the present study a significant correlation between FDG uptake and the TRG system for HCRC was found $(p=0.001)$. A cut off at $-41 \%$ dSUV for responding lesions could be identified resulting in a positive predictive value of over $83 \%$. Therefore, similar to the results for locally advanced rectal cancer the requested decrease in $\mathrm{SUV}_{\max }$ after neoadjuvant chemotherapy might be higher for HCRC than the currently generally recommended $15-30 \%$ to reflect histopathological tumor regression $[10,13]$. This underlines the need to determine PET-response criteria individually for each cancer entity as it has been demonstrated for esophageal cancer as well $[11,12]$. Furthermore, an increase in dSUV was predictive for histopathological non-response in all cases.

FDG PET/CT cannot replace a histopathological work up after tumor resection. However, preoperative FDG PET has shown to detect distant recurrences and is therefore an efficient tool in the selection of resectable patients after neo-adjuvant chemotherapy [5]. Additionally, reliable noninvasive assessment of treatment efficacy can be helpful for the oncologist for further treatment planning of the patients, since the same chemotherapy regimen can be continued, e.g., in difficult surgical situations when complete resection in a single step procedure is not possible or for completion of neo-adjuvant treatment. 

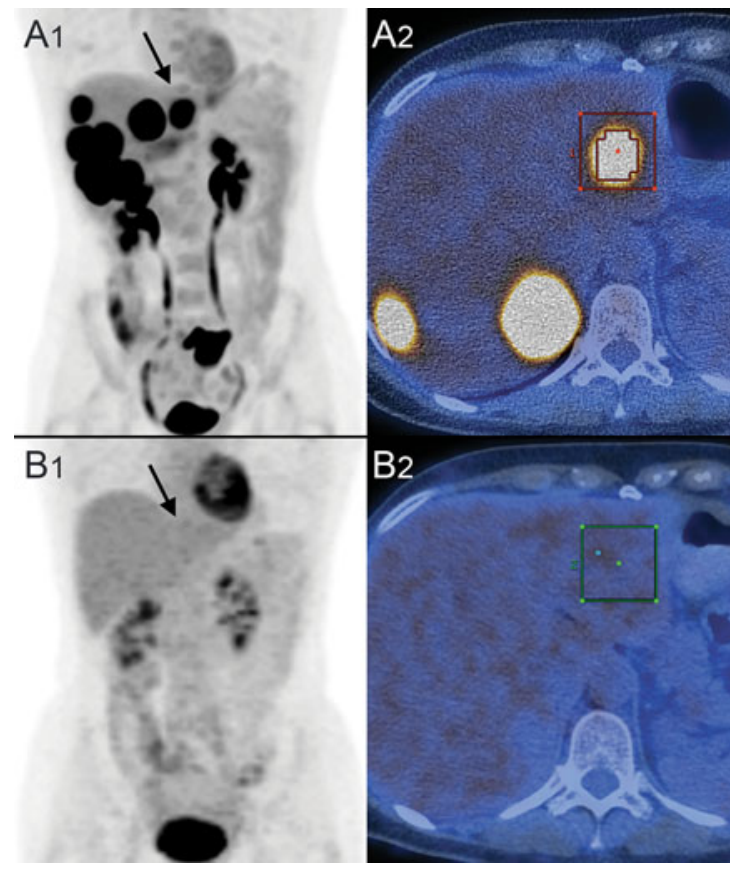

B2
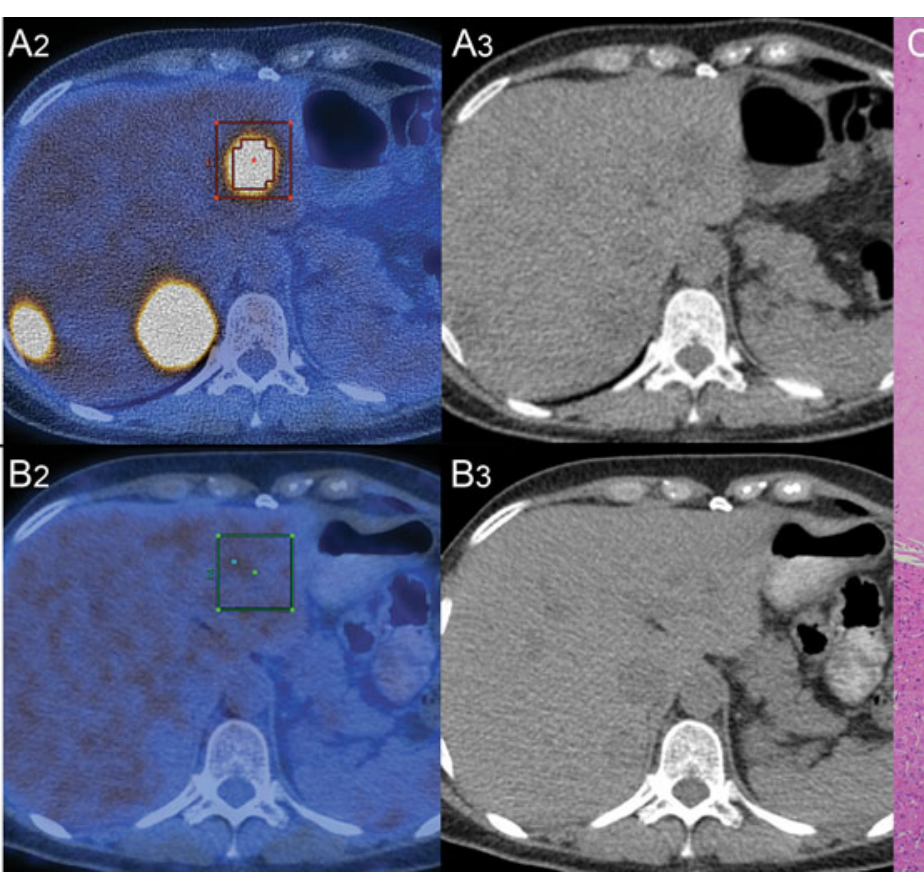

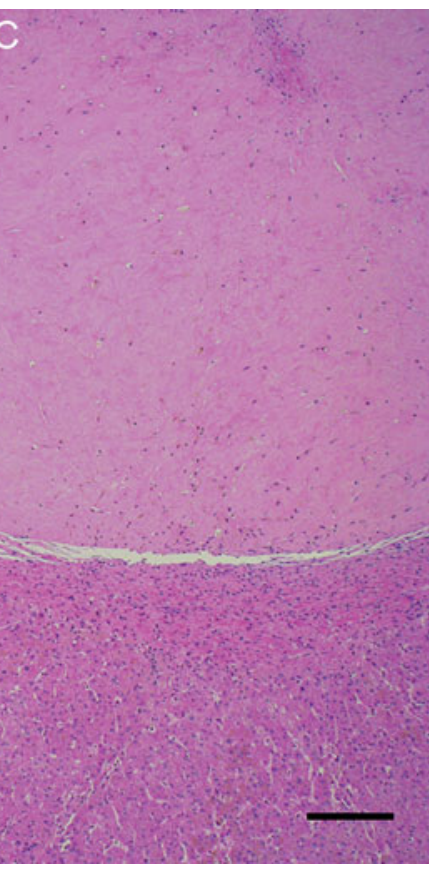

Fig. 4 51-year-old woman with hepatic metastases of a sigma carcinoma pT4 N1 M1. $\mathbf{a}_{1-3}$ Extensive FDG active liver metastases in both lobes in the initial PET/CT (18 June 2009) the lesion in liver segment II was selected (arrow). (a $\mathbf{a}_{1}$ ) The lesion was well deliniated on axial PET/CT images $\left(\mathbf{a}_{2}\right)$, but not well demarcated on axial unenhanced

Table 3 TRG versus FDG PET/CT

\begin{tabular}{lccc}
\hline $\begin{array}{l}\text { Cut off for dSUV } \\
(-41 \%)\end{array}$ & $\begin{array}{l}\text { TRG } \\
\text { responding }\end{array}$ & $\begin{array}{l}\text { TRG non- } \\
\text { responding }\end{array}$ & \\
\hline FDG responding & 15 & 3 & 18 \\
FDG non-responding & 5 & 8 & 13 \\
& 20 & 11 & 31
\end{tabular}

Sensitivity $72 \%$, Specificity $75 \%$, Negative predictive value $62 \%$, Positive predictive value $83 \%$

Accuracy $74 \%$

A limitation of the presented data is the retrospective nature, which makes an accurate identification and correlation of lesions in pathology reports with the FDG PET/ CT images difficult. We were therefore limited to 31 lesions. This also limited the possibility for outcome analysis; since only 23 patients were included we focused on the lesion-based analysis only.

Larger studies analyzing the correlation between change in SUV and histopathological tumor response are desirable, ideally in a prospective setting with response assessment scheduled within a standardized timeframe after completion of neoadjuvant therapy and imaging protocols [22]. In the present study, the variable time between chemotherapy and surgery among different patients limits the informative
CT $\left(\mathbf{a}_{3}\right) \cdot \mathbf{b}_{1-3}$ After 8 cycles of FOLFIRI with Avastin (until the 8 October 2009) the SUV uptake decreased completely (dSUV -79 \%) in the second PET (5 November 2009) ( $\left.\mathbf{b}_{\mathbf{2}}\right)$. c Histopathology of the lesion in segment II (arrow) revealed extensive fibrosis (95\%) corresponding to TRG 1 (H\&E stain, scale bar $200 \mu \mathrm{m})$

value of the histopathological response scoring. However, this was still in accordance with the retrospective manner and selection of patients with different therapies and time regimes that were used to predict pathological response [16]. Also the chosen time frame of 8 weeks between PET and surgery is within the range of previously published studies for FDG PET/CT response assessment for HCRC [18]. A further limitation is that we did not have contrast enhanced CT-scans. Therefore, morphologic response assessment was not performed and a comparison of metabolic versus morphologic response not possible.

To strengthen or modify the result of $41 \%$ decrease in FDG uptake presented in this study, larger cohorts imaged with strictly defined protocols will be required. However, these are the first results analyzing the correlation between FDG decrease and TRG for HCRC and they suggest that we might need to reevaluate the partial metabolic response criterion in FDG PET/CT therapy response assessment and seek individual thresholds for different tumors.

\section{Conclusion}

A relative change in FDG activity of more than $41 \%$ can be a prognostic tool to predict histopathological response to chemotherapy in HCRC. A relative increase or a low-to- 


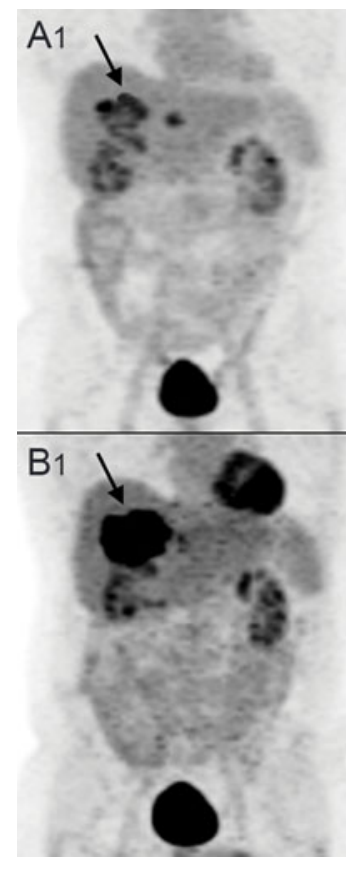

Fig. 5 54-year-old man with hepatic metastases of a colon carcinoma pT3 N2 M1. $\mathbf{a}_{1-3}$ The initial PET/CT (27 August 2009) revealed three FDG active liver metastases, the largest in segment IVa (arrow). $\mathbf{b}_{1-3}$ After 5 doses of FOLFIRI (until 1 December 2009) the

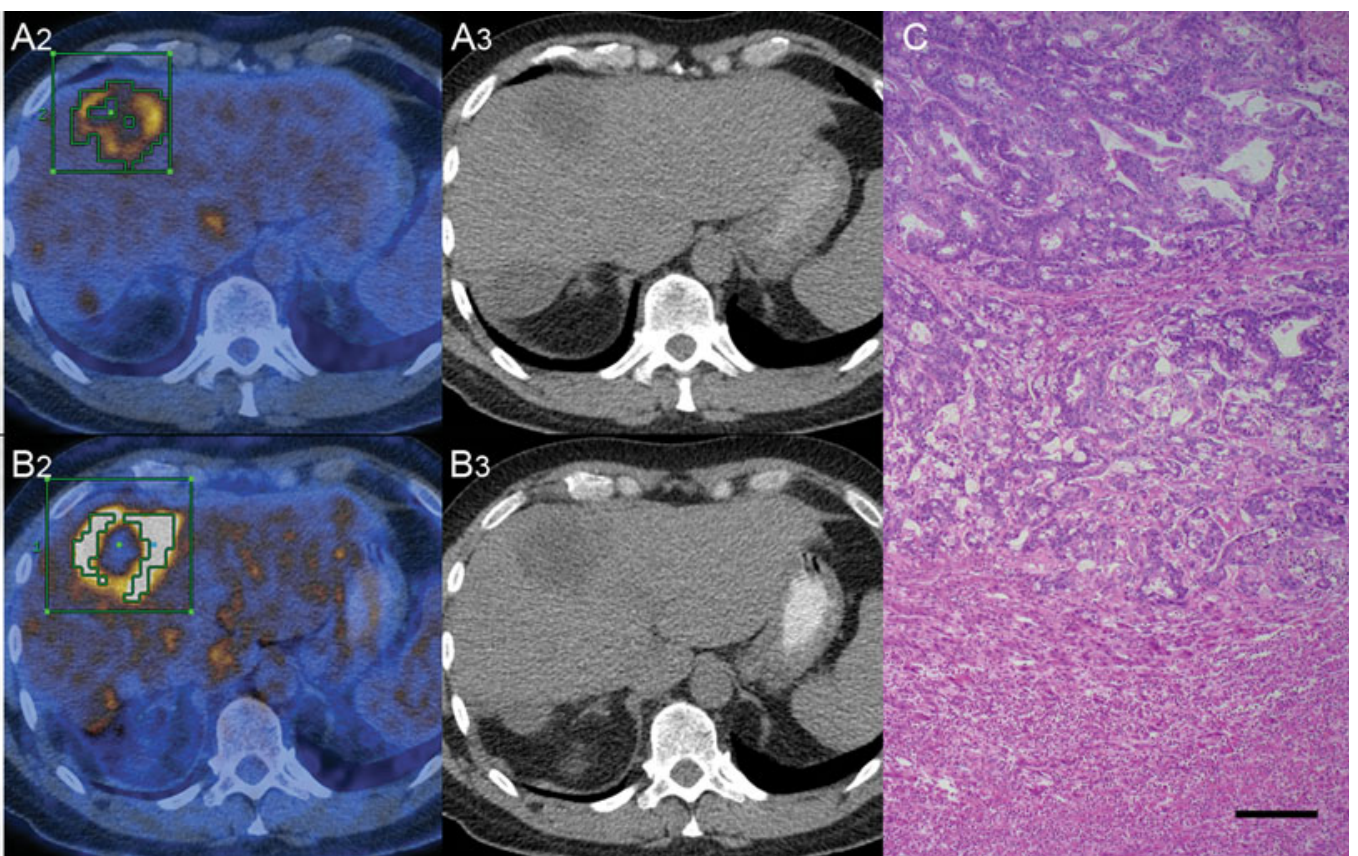

SUV uptake increased (dSUV $+126 \%$ ) in the second PET (18

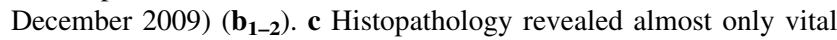
tumor cells in the metastasis in segment IVa (95\%) corresponding to TRG 5 (H\&E stain, scale bar $200 \mu \mathrm{m}$ )
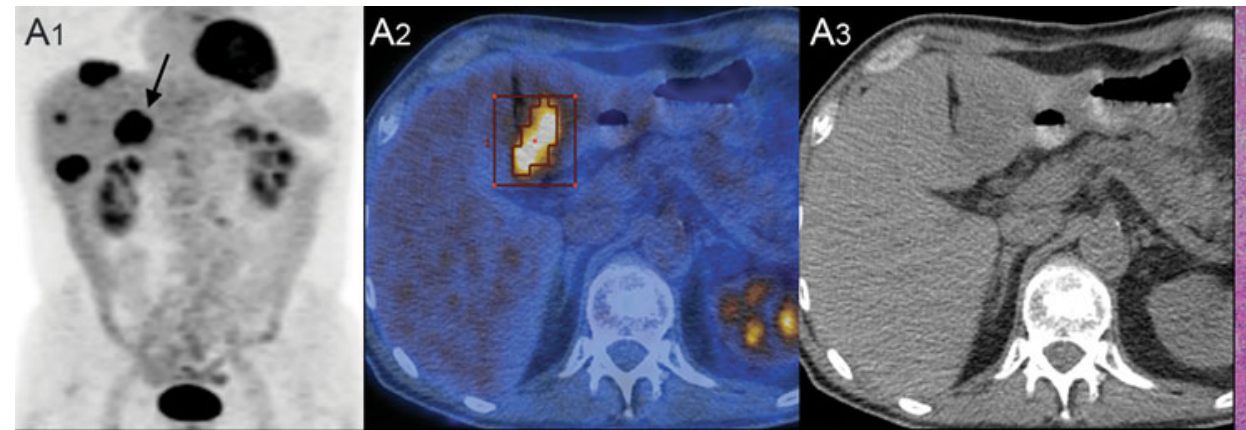

$\mathrm{C}_{1}$
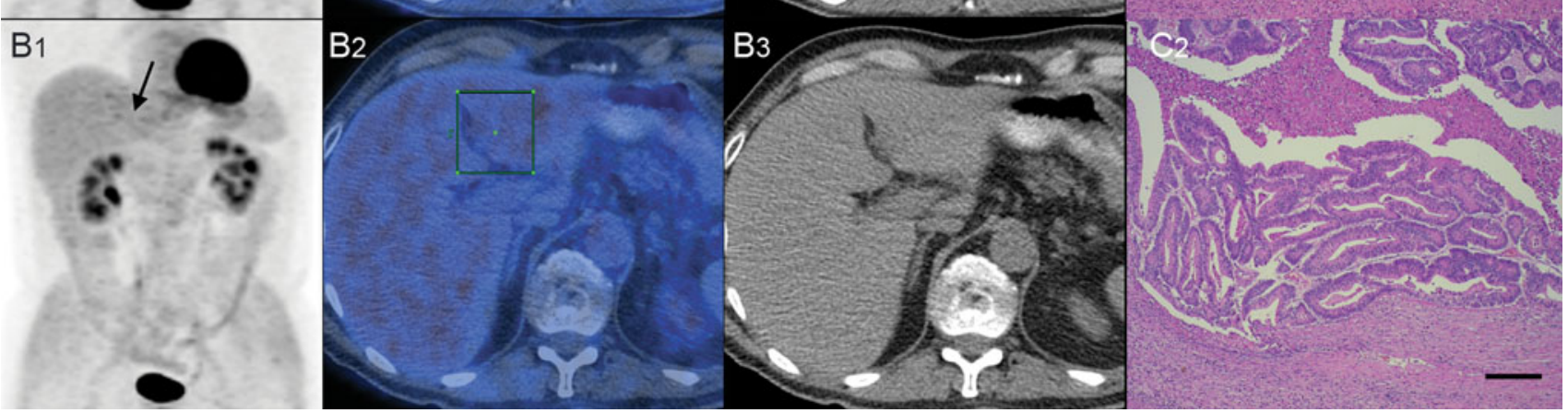

Fig. 6 55-year-old man with hepatic metastases of colon carcinoma pT3 N2 M1. a 1-3 Initial PET/CT (7 August 2009) with five FDG active liver metastases - the lesion in segment III (arrow) was further analysed. $\mathbf{b}_{\mathbf{1 - 3}}$ After 6 cycles of FOLFOX with Avastin (until 6 October 2009) the SUV uptake decreased significantly (dSUV
$-76 \%$ ) (Second PET, 28 October 2009). $\mathbf{c}_{\mathbf{1 - 2}}$ Histopathology of segment III revealed a mixed pattern, with extensive necrosis

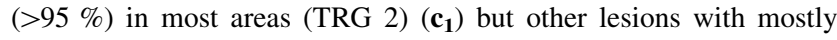
vital tumor cells $\left(\mathbf{c}_{2}\right)$, therefore resulting in TRG 4 (H\&E stain, scale bar $200 \mu \mathrm{m})$ 
moderate decrease in SUV indicated that hepatic lesions did not respond to therapy.

\section{References}

1. Lordick F, Ruers T, Aust DE, Collette L, Downey RJ, El Hajjam $\mathrm{M}$, et al. European Organisation of Research and Treatment of Cancer (EORTC) Gastrointestinal Group: Workshop on the role of metabolic imaging in the neoadjuvant treatment of gastrointestinal cancer. Eur J Cancer. 2008;44:1807-19.

2. Venook A. Critical evaluation of current treatments in metastatic colorectal cancer. Oncologist. 2005;10:250-61.

3. Adam R, Delvart V, Pascal G, Valeanu A, Castaing D, Azoulay $\mathrm{D}$, et al. Rescue surgery for unresectable colorectal liver metastases downstaged by chemotherapy: a model to predict long-term survival. Ann Surg. 2004;240:644-57.

4. Alberts SR, Horvath WL, Sternfeld WC, Goldberg RM, Mahoney MR, Dakhil SR, et al. Oxaliplatin, fluorouracil, and leucovorin for patients with unresectable liver-only metastases from colorectal cancer: a North Central Cancer Treatment Group phase II study. J Clin Oncol. 2005;23:9243-9.

5. Ruers TJ, Wiering B, van der Sijp JR, Roumen RM, de Jong KP, Comans EF, et al. Improved selection of patients for hepatic surgery of colorectal liver metastases with (18)F-FDG PET: a randomized study. J Nucl Med. 2009;50:1036-41.

6. Monteil J, Mahmoudi N, Leobon S, Roudaut PY, El Badaoui A, Verbeke S, et al. Chemotherapy response evaluation in metastatic colorectal cancer with FDG PET/CT and CT scans. Anticancer Res. 2009;29:2563-8.

7. de Geus-Oei LF, van Laarhoven HW, Visser EP, Hermsen R, van Hoorn BA, Kamm YJ, et al. Chemotherapy response evaluation with FDG-PET in patients with colorectal cancer. Ann Oncol. 2008;19:348-52.

8. Young H, Baum R, Cremerius U, Herholz K, Hoekstra O, Lammertsma AA, et al. Measurement of clinical and subclinical tumour response using [18F]-fluorodeoxyglucose and positron emission tomography: review and 1999 EORTC recommendations. European Organization for Research and Treatment of Cancer (EORTC) PET Study Group. Eur J Cancer. 1999;35: 1773-82.

9. Wahl RL, Jacene H, Kasamon Y, Lodge MA. From RECIST to PERCIST: evolving considerations for PET response criteria in solid tumors. J Nucl Med. 2009;50.

10. Capirci C, Rampin L, Erba PA, Galeotti F, Crepaldi G, Banti E, et al. Sequential FDG-PET/CT reliably predicts response of locally advanced rectal cancer to neo-adjuvant chemo-radiation therapy. Eur J Nucl Med Mol Imaging. 2007;34:1583-93.
11. Gillham CM, Lucey JA, Keogan M, Duffy GJ, Malik V, Raouf AA, et al. (18)FDG uptake during induction chemoradiation for oesophageal cancer fails to predict histomorphological tumour response. Br J Cancer. 2006;95:1174-9.

12. Klaeser B, Nitzsche E, Schuller JC, Koberle D, Widmer L, Balmer-Majno S, et al. Limited predictive value of FDG-PET for response assessment in the preoperative treatment of esophageal cancer: results of a prospective multi-center trial (SAKK 75/02). Onkologie. 2009;32:724-30.

13. Palma P, Conde-Muino R, Rodriguez-Fernandez A, SeguraJimenez I, Sanchez-Sanchez R, Martin-Cano J, et al. The value of metabolic imaging to predict tumour response after chemoradiation in locally advanced rectal cancer. Radiat Oncol. 2010;5:119.

14. Lordick F, Ott K, Krause BJ, Weber WA, Becker K, Stein HJ, et al. PET to assess early metabolic response and to guide treatment of adenocarcinoma of the oesophagogastric junction: the MUNICON phase II trial. Lancet Oncol. 2007;8:797-805.

15. Mandard AM, Dalibard F, Mandard JC, Marnay J, Henry-Amar $\mathrm{M}$, Petiot JF, et al. Pathologic assessment of tumor regression after preoperative chemoradiotherapy of esophageal carcinoma. Cancer. 1994;73:2680-6.

16. Rubbia-Brandt L, Giostra E, Brezault C, Roth AD, Andres A, Audard $\mathrm{V}$, et al. Importance of histological tumor response assessment in predicting the outcome in patients with colorectal liver metastases treated with neo-adjuvant chemotherapy followed by liver surgery. Ann Oncol. 2007;18:299-304.

17. Calvo FA, Domper M, Matute R, Martinez-Lazaro R, Arranz JA, Desco M, et al. 18F-FDG positron emission tomography staging and restaging in rectal cancer treated with preoperative chemoradiation. Int J Radiat Oncol Biol Phys. 2004;58:528-35.

18. Capirci C, Rubello D, Chierichetti F, Crepaldi G, Fanti S, Mandoliti $\mathrm{G}$, et al. Long-term prognostic value of 18F-FDG PET in patients with locally advanced rectal cancer previously treated with neoadjuvant radiochemotherapy. AJR Am J Roentgenol. 2006; 187:W202-8.

19. von Schulthess GK, Steinert HC, Hany TF. Integrated PET/CT: current applications and future directions. Radiology. 2006;238:405-22.

20. Fluss R, Faraggi D, Reiser B. Estimation of the Youden Index and its associated cutoff point. Biom J. 2005;47:458-72.

21. Findlay M, Young H, Cunningham D, Iveson A, Cronin B, Hickish T, et al. Noninvasive monitoring of tumor metabolism using fluorodeoxyglucose and positron emission tomography in colorectal cancer liver metastases: correlation with tumor response to fluorouracil. J Clin Oncol. 1996;14:700-8.

22. Shankar LK, Hoffman JM, Bacharach S, Graham MM, Karp J, Lammertsma AA, et al. Consensus recommendations for the use of $18 \mathrm{~F}-\mathrm{FDG}$ PET as an indicator of therapeutic response in patients in National Cancer Institute Trials. J Nucl Med. 2006;47:1059-66. 